\title{
La cámara como principal herramienta para la investigación audiovisual de los procesos de dinamización intercultural mediatizados
}

\section{Nicolás lorite garcía}

Doutor; Universitat Autònoma de Barcelona

nicolas.lorite@uab.cat

\section{Resumen}

Este texto va destinado a mostrar diferentes ideas sobre la cámara como principal herramienta metodológica para investigar el tratamiento mediático de la realidad y en concreto la re-presentación social en los medios de comunicación, realizadas desde el MIGRACOM (Observatorio y Grupo de Investigación de Migración y Comunicación) de la Universidad Autónoma de Barcelona (UAB), durante las dos últimas décadas (1996-2015). Se usa la cámara a partir del estudio interdisciplinar (comunicación, antropología, pedagogía, política, sociología y lingüística) del mismo objeto de estudio audiovisual y la multimodalidad metodológica o análisis transversal desde la producción, emisión, recepción y dinamización de los mensajes audiovisuales. Asimismo, la cámara permite vincular la investigación científica, académica, con la producción audiovisual real. Su uso emerge del mestizaje de la racionalidad cuantitativa con la espontaneidad cotidiana.

\section{Palabras clave}

Cámara. Audiovisual. Televisión. Multimodalidad. Interculturalidad.

\section{Introducción}

Desde que vengo impartiendo lenguaje audiovisual a principios de los 90 en la universidad a futuros periodistas, publicitarios y comunicadores audiovisuales, he defendido que el análisis del tratamiento y representación de la sociedad en los medios audiovisuales es conveniente llevarlo a cabo desde una perspectiva audiovisual también, consistente en ana- 
lizar el audiovisual desde el audiovisual y hacerlo desde la mirada de la cámara y no desde el pensamiento únicamente oral y textual. No hay que pensar en concluir la investigación tan sólo en un mensaje escrito (informe, libro o artículo) sino en una obra audiovisual que proponga, de manera audiovisual también, lo analizado y criticado de la producción audiovisual. Desde que llevo defendiendo esta idea he podido constatar que somos excelentes analistas y críticos televisivos y cinematográficos pero pésimos productores de la alternativa audiovisual apropiada. Analizamos los contenidos televisivos, publicitarios y cinematográficos desde un pensamiento bastante crítico, a veces demasiado crítico según la ideología de referencia, que deriva hacia la argumentación oral o textual pero no hacía la audiovisual. Se critica una imagen pero no se ofrece otra imagen como alternativa. Es por ello que siempre he procurado, como investigador audiovisual (pido disculpas por redundar en esta palabra en lo poco escrito en este largo párrafo y en lo que vendrá a continuación) huir del sobrevalorado reduccionismo textual y proponer, aunque soy consciente que en parte de manera utópica, que la obra audiovisual puede albergar el mismo valor científico que la textual y que su transferencia en la comunidad científica y en la sociedad en general, debe ser evaluada con los mismos indicadores científicos. Resulta algo paradójico observar como en un mundo tan audiovisual como el actual es obligatorio aún sintetizar las conclusiones de una investigación audiovisual, editadas en un documental, en artículos escritos, a ser posible en alguna revista prestigiosa en inglés, para lograr el oportuno reconocimiento de la comunidad científica.

A continuación se exponen algunas ideas, surgidas de la experimentación investigadora audiovisual de 20 años, que permiten mostrar cómo es posible incorporar la cámara (desde la perspectiva audio y/o visual), tanto como principal herramienta de análisis de los mensajes audiovisuales como principal instrumento para producir y mostrar el resultado de la investigación de manera audiovisual también, partiendo de ciertos postulados científicos de referencia obligada como los del rigor científico (CHALMERS, 2000). No se trata de un uso gratuito o improvisado de la cámara. Dicho uso, vinculado con el rigor, emana del contraste teórico y metodológico al investigar la representación audiovisual de la sociedad de manera interdisciplinar (comunicación, pedagogía, lingüística, antropología, sociología y política principalmente), y hacerlo, además, desde la multimodalidad metodológica que supone analizar los discursos audiovisuales desde la producción, emisión, recepción y dinamización social, pensando en la incorporación de la cámara en cada uno de estos cuatro ámbitos, y en la conexión de dicho proceso con la producción audiovisual real, en concreto con 
las rutinas productivas y las guías de recomendaciones y manuales de estilo de los medios de comunicación y sus organizaciones. Con esto la cámara no sólo pretende ser una herramienta metodológica científica sino un puente que permita conectar la Academia con la realidad productiva audiovisual (LORITE-GARCÍA, 2006a, 2006b, 2007a, 2008, 2011).

\section{Acerca del paradigma científico del audiovisual}

Una primera cuestión que preocupa sobre el uso de la cámara es la de otorgarle el correspondiente rigor científico a la investigación audiovisual. Cómo estamos empeñados en demostrar a la comunidad científica que la cámara puede hacer ciencia hay que demostrar que se diseñan los planteamientos iniciales desde el método científico: se concreta el objeto de estudio y se perfila el primer planteamiento hipotético y la metodología cuanticualitativa. En este texto sólo se recoge de manera muy esquemática algunos autores a los que hemos ido acudiendo para defender el rigor científico con la cámara. Autores como Chalmers (2000) e Ibañez (1979) ayudan a comprender que puede otorgársele el calificativo científico a cualquier proceso investigador siempre y cuando sea posible argumentar y defender los principios en los que se basa. También es posible encontrar bastantes referencias sobre la relatividad del proceder científico desde el mestizaje etnográfico de la antropología con el visual en especialistas que se han enfrentado a este problema, como Achutti (1997), y que defienden la fotoetnografía como científica, o lo que recogen Canevacci (2001) y Grau Rebollo (2000; 2003) desde la cámara como sustituta del cuaderno de notas desde la antropología audiovisual. Sin perder de vista las aportaciones de Berger (1997, 2000 y 2001) que nos muestra esa necesidad de usar la cámara desde la doble óptica del mirar y el ser mirado, las de Berger y Luckmann (1984) sobre la construcción social de la realidad y cómo se fragua la mirada del individuo, y a partir de ahí su mirada través de la cámara, durante su socialización primaria, llevada a cabo durante la infancia, o bien las de la escuela de Palo Alto sobre la representación subjetiva de la realidad (WATZLAWICK, 1995).

Uno de los objetivos principales del uso científico audiovisual de la cámara es el de la presentación de las conclusiones mediante un discurso, obra o documental que aborde los temas investigados mediante la teoría del montaje audiovisual que entrelace los materiales codificados en la base de datos con el mayor número posible de opiniones contrastadas de los principales actores implicados en la representación mediática de la sociedad. Así se llegó a producir el documental "En torno a la muerte de Ousmane Kote" para mostrar el resultado del análisis del tratamiento informativo de la inmigración en España. Se selecciona uno de 
los casos que ha producido más noticias en el 2008 y se aborda desde las opiniones de los periodistas, técnicos de la inmigración, políticos, protagonistas y demás actores implicados en la producción y recepción de la noticia.

La escaleta y guion de dicha obra audiovisual se concibe desde los primeros pasos embrionarios de la investigación, desde el debate inicial sobre el método científico adecuado para llevarla a cabo. Se trata, por tanto, de realizar un discurso audiovisual que incorpora, tanto en su diseño inicial como en su edición final, los planteamientos epistemológicos, teóricos y metodológicos propios de cualquier investigación (LORITE-GARCÍA; GRAU REBOLLO, 2013; LORITE-GARCÍA, 2006a, 2006b, 2007a, 2011). Se piensa en la cámara al diseñar la hipótesis principal, el marco teórico con los conceptos principales de referencia para llevar a cabo el análisis y la producción audiovisual, y la metodología cuanti-cualitativa (por este orden, antes cuanti que cuali, que permita ir de una muestra cuantitativa objetiva, codificada a partir de variables independientes al cualitativo posterior que permita profundizar en la especificidad temática y en la obtención del caso).

En cuanto a la consideración científica del audiovisual, pensado desde el soporte de la cámara como herramienta metodológica principal, cabe ampararse en autores como Kuhn (1975) y en lo que considera sobre los paradigmas como los procesos más adecuados desde el momento que el contraste entre la delimitación de los problemas y su resolución deriva en propuestas científicas universales. Apostamos por un paradigma científico propio del audiovisual capaz de albergar problemas y soluciones sobre el estudio de la representación audiovisual de la sociedad en los medios de comunicación e información, con el correspondiente abanico de planteamientos epistemológicos, teóricos y metodológicos, lo más abierto posible, que permita otorgarle el máximo rigor científico a nuestra perspectiva audiovisual, en una comunidad científica a la que le cuesta incorporarlo como ciencia, partiendo siempre de la convicción de que los paradigmas no son estáticos y se re-construyen permanentemente a partir del falsacionismo que sugiere Popper (1962), e incluso ganan cientificidad al circular transitoriamente por la ruptura epistemológica que propone Feyarabend (1984). Aun así, después de experimentar dichos procesos, tenemos claro que no pretendemos finalizar en la anarquía epistemológica sino en un mestizaje heurístico del pensamiento audio y/o visual con el racional oral-textual. Hay que considerar dicho rigor científico audiovisual como suma de ambos. No hay que ser tan sólo textual pero tampoco antitextual en esta fase de tímida aceptación del audiovisual por parte de la Academia. Amplia los puntos de mira redactar un texto como éste defendiendo la cámara y el documental como la mejor manera de 
mostrar científicamente los resultados de una investigación audiovisual aunque lo ideal sería difundir el documental únicamente.

A todo ello hay que agregarle la siguiente observación: es fácil criticar, por lo general de manera oral desde el sofá, el tratamiento racista, xenófobo y discriminador de los colectivos inmigrantes en la televisión, pero ya no resulta tan fácil proponer una alternativa audiovisual, un tratamiento televisivo adecuado. Dicha propuesta pasa por agregarle a la opinión, principalmente oral, que somos capaces de argumentar al visionar cualquier noticia televisiva, el tratamiento visual y sonoro adecuado. A veces se comenta lo que se oye y lee en los titulares de la notica televisiva pero no lo que se ve, otras se opina sobre una imagen fija o en movimiento impactantes, pero pocas veces se agrega a dicha crítica oral la aportación audiovisual constructiva. Las audiencias son tan orales y textuales como la Academia y no están instruidas para proponer el mensaje alternativo. Se enseña a escribir y a leer textos pero no a leer obras audiovisuales con la gramática audiovisual. Hay un desconocimiento absoluto del lenguaje audiovisual y de las complejas rutinas que comporta cualquier producción audiovisual o realización televisiva. Por tanto, la obra audiovisual final de cualquier proceso de investigación audiovisual debe reunir este plus de propuesta alternativa pensando en la formación de su audiencia potencial. El visionado del documental, que emana de la investigación audiovisual, pretende denotar que es científico por sí mismo al ser visto pero también está pensado para otorgar a los receptores del mensaje los elementos oportunos del lenguaje audiovisual para plantear la crítica constructiva audiovisual y propuesta alternativa audiovisual adecuada, que incluso sea fácil de plasmar por los profesionales del audiovisual, para re-presentar a la sociedad en los medios de comunicación (LORITEGARCÍA, 2006a, 2006b).

\section{Perspectivas intrínsecas y extrínsecas del audiovisual}

Una segunda cuestión que nos preocupa, desde los diferentes proyectos del MIGRACOM (Observatorio y Grupo de Investigación de Migración y Comunicación de la Universidad Autónoma de Barcelona), es la del uso de la cámara, no sólo como herramienta metodológica principal para el estudio de la representación mediática de la sociedad, sino para analizar e intervenir en los procesos de dinamización intercultural desde la doble perspectiva intrínseca y extrínseca del audiovisual. Consideramos perspectivas audiovisuales intrínsecas los lenguajes fotográficos, musicales, publicitarios, televisivos y cinematográficos, y extrínsecas las que se realizan desde otros ámbitos, colaterales al audiovisual, y con los que 
venimos trabajando conjuntamente de manera interdisciplinar, como la antropología, la pedagogía, el lenguaje y la política principalmente.

Desde la perspectiva intrínseca obtenemos indicadores y datos extrapolables objetivos que permiten proponer, conjuntamente con los profesionales que trabajan a diario en las rutinas productivas de los medios de comunicación e información, así como con sus órganos colegiados (colegios de periodistas, colegios de publicitarios, consejos del audiovisual, observatorios del racismo y la xenofobia, etc.), y demás instituciones políticas y organizaciones sociales, los discursos audiovisuales, adecuados y éticos, dinamizadores de las relaciones interculturales, y potenciadores del respeto a la diversidad sociocultural. Dicha perspectiva de investigación audiovisual aplicada la ponemos en práctica desde la primera investigación del año 1996, llevada a cabo conjuntamente con el departamento de Bienestar Social del Gobierno de Cataluña o Generalitat de Catalunya, y la organización de periodistas Colegio de Periodistas de Catalunya. De este proyecto conjunto de investigación audiovisual entre universidad y realidad periodística, política y económica, emergen propuestas como la del Manual de Estilo sobre Inmigración y Minorías Étnicas, primero en proponerse en España y uno de los primeros de Europa y antesala del "Manual sobre el tratamiento informativo de la inmigración en los medios de comunicación" del Consejo del Audiovisual de Cataluña del 2002 (CONSELL DE L'AUDIOVISUAL DE CATALUNYA, 2004).

Dicha dinámica de investigación audiovisual aplicada se extiende del ámbito catalán (LORITE-GARCÍA, 1996) al español con los proyectos sobre el Tratamiento Informativo de la Inmigración en España, realizados desde el año 2002 hasta el 2010 por el MIGRACOM-UAB con el OBERAXE (Observatorio del Racismo y la Xenofobia Español), perteneciente a la Secretaría General de la Emigración y Emigración, Dirección General de Integración de los Inmigrantes del Ministerio de Trabajo e Inmigración (LORITE-GARCÍA, 2004, 2006a, 2006b, 2007a, 2007b, 2008a, 2008b, 2009, 2010). Mediante dichos trabajos se profundiza en cuál debe ser la mirada audiovisual más adecuada de la diversidad sociocultural y la inmigración desde la conjunción del análisis y grabación audio y/o visual con las recomendaciones audiovisuales a los medios de comunicación como las que recoge la "Guía Práctica para los Profesionales de los Medios de Comunicación. Tratamiento Mediático de la Inmigración" (SENDÍN; IZQUIERDO, 2008).

Una perspectiva intrínseca al audiovisual es la imagen, en particular el análisis del uso pertinente que hacen de la fotografía en la prensa escrita y de la imagen fija y en movimiento en la televisión (BERTRAN, 2003; LORITE-GARCÍA, 2004, 2006a, 2006b, 2007a, 2008a, 
2008b, 2010). Desde el primer estudio del año 1996 se han obtenido ciertos indicadores objetivos que permiten constatar que se está dando un tratamiento visual mediático discriminatorio a los colectivos inmigrantes, venidos a España de África, Latinoamérica y Asia. Para verificar dicha discriminación visual, que puede derivar, a su vez, en efectos racistas y xenófobos, partimos del análisis cuantitativo o selección de muestras objetivas anuales de los principales diarios y telenoticiarios de ámbito estatal y regional del estado español.

Un primer indicador obvio del tratamiento visual consiste en verificar si aparecen inmigrantes en las imágenes que abordan noticias sobre ellos, y, en caso de aparecer, comprobar si se les trata de manera individual o en grupo y que composición visual o planos se usan principalmente para mostrarlos. Es importante comprobar que tipo de plano que se usa, en concreto si los muestran en primer plano, plano medio o plano general, y el valor del plano: el primer plano lo aproxima, el plano general lo aleja. También comprobamos si el punto de vista con la cámara es frontal, y por tanto insinúa un tratamiento igualitario del inmigrante con el telespectador, o si lo muestran en picado, o toma estática con la cámara de arriba a abajo, y lo trata como inferior; o bien, al contrario, toma estática en contrapicado, de abajo arriba, y, por tanto, lo ensalza (GUBERN, 1987, p. 271). El comparativo visual es fundamental. Comprobamos si los autóctonos son tratados de la misma manera que los inmigrantes. Habitualmente el autóctono, y en particular la fuente oficial, en particular los responsables políticos y demás autoridades o responsables de organizaciones, es tratada en plano medio frontal o composición que ubica al personaje en la proximidad de una conversación familiar con el receptor. Tomamos como referencia dichos principios del lenguaje cinematográfico que ha tomado el lenguaje televisivo y audiovisual en general de directores como Eisenstein y películas como el Acorazado Potenkin (ANDREW, 1978). A estos diferentes criterios de la composición visual cabe agregar otros como el entorno o lugar mostrado, la actitud de los protagonistas, el tema abordado, etc. El tratamiento inapropiado y discriminador es aquel que muestra la heroicidad de la llegada de los inmigrantes a la Europa prometida en plano general, de noche, en picado, en un entorno hostil que lo recibe con la policía (BERTRAN, 2003; LORITE-GARCÍA, 2008b).

Otra perspectiva investigadora intrínseca fundamental es la del discurso sonoro y en particular el lenguaje musical. Hay que analizar algo tan sumamente concreto pero impactante como son las músicas utilizadas en las sintonías de las caretas de presentación de los telenoticiarios de mayor audiencia, para comprender si detentan una identidad cultural plural, y pueden ser decodificadas de la misma manera por todos los telespectadores o re- 
ceptores del mensaje, indistintamente de su procedencia geográfica y adscripción sociocultural, o sólo representan a una parte de la sociedad autóctona. Eduardo Muraca diseña unos primeros indicadores en este sentido para la investigación "Tratamiento de la Inmigración en España, año 2007" (LORITE-GARCÍA, 2007b) que delimita con su tesina sobre los procesos comunicativos de la música (MURACA, 2009) y acaba validando con su tesis doctoral Diseño y comunicación de la pluralidad sociocultural en los discursos musicales de los teleinformativos (MURACA, 2013). En concreto, analiza una muestra de los discursos sonoros emitidos por las sintonías musicales de las caretas de presentación de los telenoticiarios de las televisiones estatales (TVE, Tele5, Antena3, Cuatro y La Sexta) y autonómicas (TV3, Canal 9, Canal Sur, TVG, Tele Madrid y ETB) y profundiza posteriormente sus diseños y efectos desde el análisis multimodal (producción, emisión, recepción y dinamización). Verifica que se trata de músicas de corte militar, centroeuropeas, originarias del siglo XVIII, y que se han usado posteriormente en el cine, en particular en películas americanas de acción de los 70. Muraca no se queda en el simple análisis musical. Produce diferentes músicas alternativas y sugiere una sintonía musical adecuada para comunicar concepciones asociadas a la diversidad sociocultural e interculturalidad de la realidad española actual. Asimismo, dicho proceso investigador le permite diseñar la música adecuada para el documental En torno a la muerte de Ousmane Kote (LORITE-GARCÍA; GRAU REBOLLO, 2013) como producto audiovisual científico del estudio del tratamiento informativo de la inmigración en España.

\section{Una obra audiovisual científica: "En torno a la muerte de Ousmane Ko- te"}

¿Cómo se produce una obra audiovisual científica a partir de una investigación audiovisual con la cámara? Ante todo vamos del cuantitativo al cualitativo, tras pasar por algunas de las reflexiones epistemológicas y teóricas, argumentadas anteriormente. Desde la perspectiva intrínseca del lenguaje informativo en la televisión hay que observar el tratamiento de la inmigración en España desde variables como: la presencia informativa o porcentaje de tiempo destinado a los colectivos inmigrantes en el total del informativo, las temáticas abordadas, la cobertura informativa, las fuentes informativas utilizadas y cuales hablan y qué dicen, el género de los protagonistas y el lenguaje audio y/o visual, extraído mediante la técnica del storyboard. Acotamos el análisis a los telenoticiarios de tarde-noche de las cadenas de televisión estatales y autonómicas durante un periodo anual de rutinas cotidianas normales (descartamos vacaciones de verano e invierno, etc.). 
La presencia informativa de la inmigración en los teleinformativos permite dimensionar el interés por la cobertura de estas informaciones sobre el total de temas abordados. $\mathrm{Si}$ tomamos como referencia las televisiones que difunden la señal a todo el estado español podemos constatar que importa la inmigración y todo lo que se deriva de ella más en los años 2002 y 2006 que en años posteriores con el inicio de la crisis económica, como podemos ver en el cuadro y gráfico siguientes.

Cuadro 1 - Presencia informativa de la inmigración en la televisión. Ámbito estatal. Período 1996 - 2010

\begin{tabular}{|c|c|c|c|c|c|}
\hline & Antena 3 & Cuatro & La Sexta & Tele 5 & TVE \\
\hline 1996 & $\begin{array}{c}0: 02: 59 \\
0,8 \% \\
\end{array}$ & - & - & $\begin{array}{c}0: 03: 36 \\
0,6 \% \\
\end{array}$ & $\begin{array}{c}0: 02: 50 \\
0,5 \% \\
\end{array}$ \\
\hline 2000 & $\begin{array}{c}0: 18: 05 \\
2,3 \%\end{array}$ & - & - & $\begin{array}{c}0: 22: 18 \\
2,9 \%\end{array}$ & $\begin{array}{c}0: 17: 06 \\
2,1 \% \\
\end{array}$ \\
\hline 2002 & $\begin{array}{c}0: 33: 20 \\
7,1 \%\end{array}$ & - & - & $\begin{array}{c}1: 06: 08 \\
9,3 \%\end{array}$ & $\begin{array}{c}0: 37: 20 \\
5,5 \% \\
\end{array}$ \\
\hline 2006 & $\begin{array}{c}2: 00: 08 \\
8,8 \% \\
\end{array}$ & $\begin{array}{c}1: 54: 37 \\
10,5 \% \\
\end{array}$ & - & $\begin{array}{c}1: 08: 00 \\
9,2 \% \\
\end{array}$ & $\begin{array}{c}2: 17: 34 \\
9,6 \% \\
\end{array}$ \\
\hline 2007 & $\begin{array}{c}1: 01: 14 \\
5,7 \% \\
\end{array}$ & $\begin{array}{c}0: 52: 58 \\
5,2 \% \\
\end{array}$ & $\begin{array}{c}0: 27: 54 \\
3,0 \% \\
\end{array}$ & $\begin{array}{c}1: 05: 27 \\
9,0 \% \\
\end{array}$ & $\begin{array}{c}0: 59: 48 \\
4,6 \%\end{array}$ \\
\hline 2008 & $\begin{array}{c}0: 39: 54 \\
4,4 \%\end{array}$ & $\begin{array}{c}0: 31: 52 \\
3,0 \%\end{array}$ & $\begin{array}{c}0: 11: 51 \\
2,9 \%\end{array}$ & $\begin{array}{c}0: 33: 09 \\
4,9 \%\end{array}$ & $\begin{array}{c}0: 25: 21 \\
3,4 \%\end{array}$ \\
\hline 2009 & $\begin{array}{c}0: 47: 11 \\
3,9 \% \\
\end{array}$ & $\begin{array}{c}0: 15: 55 \\
1,7 \% \\
\end{array}$ & $\begin{array}{c}0: 33: 25 \\
3,9 \%\end{array}$ & $\begin{array}{c}1: 11: 25 \\
5,3 \%\end{array}$ & $\begin{array}{c}0: 45: 26 \\
3,2 \%\end{array}$ \\
\hline 2010 & $\begin{array}{c}0: 43: 18 \\
3,4 \% \\
\end{array}$ & $\begin{array}{c}0: 48: 14 \\
5,4 \% \\
\end{array}$ & $\begin{array}{c}0: 46: 05 \\
4,4 \%\end{array}$ & $\begin{array}{c}1: 04: 27 \\
5,1 \% \\
\end{array}$ & $\begin{array}{c}0: 57: 12 \\
3,3 \% \\
\end{array}$ \\
\hline
\end{tabular}

Fuente: MIGRACOM (2010)

Nota: Cada cuadrícula muestra en la parte superior el total de unidades informativas, en el centro el tiempo total de voz de cada fuente informativa, medido en horas, minutos y segundos, y abajo el porcentaje de tiempo.

Gráfico 1 - Tiempo destinado a inmigración 1996-2010.

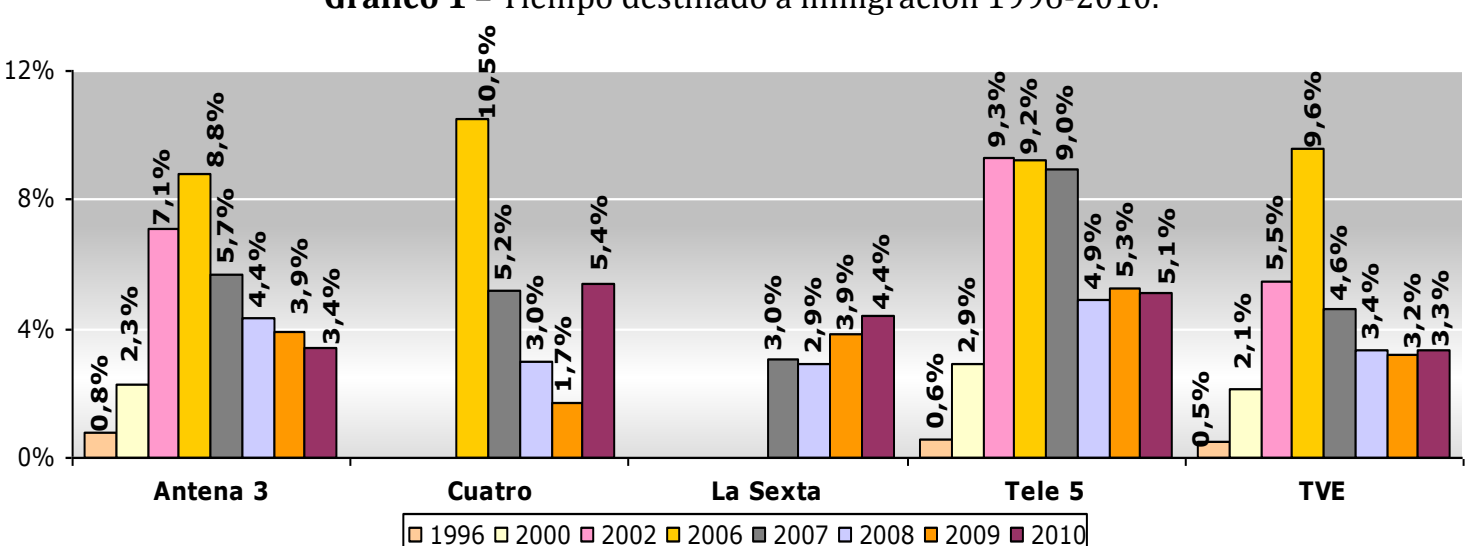

Fuente: MIGRACOM (2010) 
A partir de ahí profundizamos en ciertos aspectos intrínsecos, abordados desde una perspectiva cuanti-cualitativa también, para comprender qué tipo de fuentes informativas se usan y cuáles son las que ocupan una mayor proporción de tiempo de habla. Todas las guías y manuales recomiendan que los inmigrantes sean, lógicamente, la principal fuente informativa visual y oral de las noticias que hablan sobre ellos pero en los diferentes estudios realizados constatamos que en todas las cadenas televisivas lo son en proporciones muy inferiores a las fuentes no inmigrantes (políticas, judiciales, autóctonas, cuerpos de seguridad y expertos principalmente), según recoge el gráfico 2 y el cuadro 2 , de acuerdo a los datos obtenidos el año 2008.

Gráfica 2 - Presencia de fuentes informativas inmigrantes y no inmigrantes.

Televisón. Ámbito estatal. Año 2008.

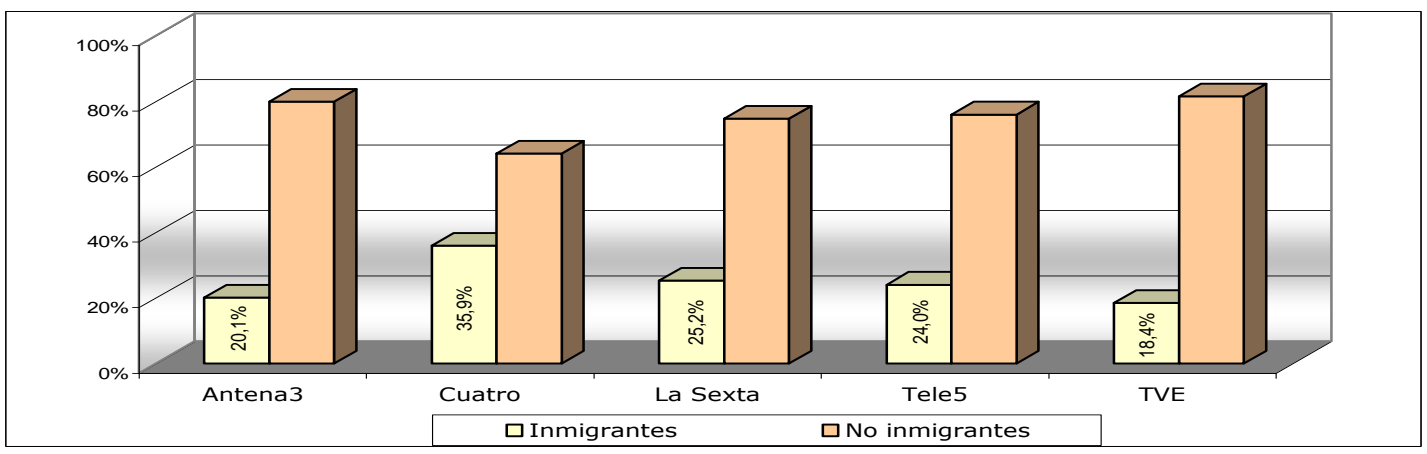

Fuente: LORITE (2011)

Cuadro 2 - Presencia de fuentes informativas. Televisión. Ámbito estatal. Año 2008.

\begin{tabular}{|c|c|c|c|c|c|c|}
\hline & Antena & Cuatro & $\underset{\text { Sexta }}{\text { La }}$ & Teles & TVE & Total \\
\hline Inm igrante & $\begin{array}{c}47 \\
0: 12: 42 \\
20.1 \% \\
\end{array}$ & $\begin{array}{c}38 \\
0: 14: 18 \\
35.9 \% \\
\end{array}$ & $\begin{array}{c}3.3 \\
0: 08: 17 \\
25.2 \%\end{array}$ & $\begin{array}{c}8.3 \\
0: 17: 40 \\
24.0 \% \\
\end{array}$ & $\begin{array}{c}5.3 \\
0: 13: 22 \\
18.4 \%\end{array}$ & $\begin{array}{c}2.54 \\
1: 06: 19 \\
23.5 \% \\
\end{array}$ \\
\hline Políticas & $\begin{array}{c}29 \\
0: 07: 27 \\
11.8 \%\end{array}$ & $\begin{array}{c}43 \\
0: 11: 30 \\
28.9 \%\end{array}$ & $\begin{array}{c}28 \\
0: 07: 15 \\
22,1 \%\end{array}$ & $\begin{array}{c}27 \\
0: 06: 44 \\
9.2 \%\end{array}$ & $\begin{array}{c}66 \\
0: 20: 04 \\
27.7 \%\end{array}$ & $\begin{array}{c}193 \\
0: 53: 00 \\
18.8 \%\end{array}$ \\
\hline Judiales & $\begin{array}{c}5 \\
0: 01: 15 \\
2,0 \% \\
\end{array}$ & $\begin{array}{c}0 \\
0: 00: 00 \\
0,0 \%\end{array}$ & $\begin{array}{c}5 \\
0: 00: 48 \\
2,4 \%\end{array}$ & $\begin{array}{c}5 \\
0: 00: 33 \\
0,7 \%\end{array}$ & $\begin{array}{c}7 \\
0: 01: 17 \\
1,8 \%\end{array}$ & $\begin{array}{c}22 \\
0: 03: 53 \\
1,4 \%\end{array}$ \\
\hline $\begin{array}{l}\text { Cuerpos de } \\
\text { seguridad }\end{array}$ & $\begin{array}{c}51 \\
0: 12: 36 \\
19,9 \% \\
\end{array}$ & $\begin{array}{c}9 \\
0: 01: 39 \\
4,1 \%\end{array}$ & $\begin{array}{c}21 \\
0: 03: 28 \\
10,6 \%\end{array}$ & $\begin{array}{c}52 \\
0: 10: 19 \\
14,0 \%\end{array}$ & $\begin{array}{c}55 \\
0: 12: 47 \\
17,6 \%\end{array}$ & $\begin{array}{c}188 \\
0: 40: 49 \\
14,5 \%\end{array}$ \\
\hline Ciudadanos & \begin{tabular}{|c|}
87 \\
$0: 21: 38$ \\
$34,2 \%$ \\
\end{tabular} & $\begin{array}{c}28 \\
0: 04: 42 \\
11,8 \%\end{array}$ & $\begin{array}{c}32 \\
0: 06: 51 \\
20,9 \%\end{array}$ & $\begin{array}{c}137 \\
0: 31: 57 \\
43,5 \%\end{array}$ & $\begin{array}{c}64 \\
0: 12: 01 \\
16,6 \%\end{array}$ & $\begin{array}{c}348 \\
1: 17: 09 \\
27,4 \%\end{array}$ \\
\hline $\begin{array}{l}\text { Movimientos } \\
\text { sociales }\end{array}$ & $\begin{array}{c}4 \\
0: 00: 38 \\
1.0 \% \\
\end{array}$ & $\begin{array}{c}15 \\
0: 01: 49 \\
4.6 \%\end{array}$ & $\begin{array}{c}7 \\
0: 01: 25 \\
4.3 \%\end{array}$ & $\begin{array}{c}7 \\
0: 00: 50 \\
1.1 \%\end{array}$ & $\begin{array}{c}13 \\
0: 01: 39 \\
2,3 \% \\
\end{array}$ & $\begin{array}{c}46 \\
0: 06: 21 \\
2,3 \%\end{array}$ \\
\hline ONGs & $\begin{array}{c}1 \\
0: 00: 17 \\
0.4 \% \\
\end{array}$ & $\begin{array}{c}4 \\
0: 00: 40 \\
1,7 \%\end{array}$ & $\begin{array}{c}3 \\
0: 00: 27 \\
1.4 \%\end{array}$ & $\begin{array}{c}2 \\
0: 00: 21 \\
0.5 \%\end{array}$ & $\begin{array}{c}3 \\
0: 00: 27 \\
0.6 \%\end{array}$ & $\begin{array}{c}13 \\
0: 02: 12 \\
0.8 \%\end{array}$ \\
\hline Expertos & $\begin{array}{c}5 \\
0: 01: 00 \\
1,6 \%\end{array}$ & $\begin{array}{c}6 \\
0: 01: 11 \\
3.0 \%\end{array}$ & $\begin{array}{c}3 \\
0: 00: 26 \\
1,3 \%\end{array}$ & $\begin{array}{c}7 \\
0: 00: 55 \\
1.2 \%\end{array}$ & $\begin{array}{c}11 \\
0: 02: 37 \\
3.6 \%\end{array}$ & $\begin{array}{c}32 \\
0: 06: 09 \\
2.2 \%\end{array}$ \\
\hline Otras & $\begin{array}{c}37 \\
0: 05: 40 \\
9.0 \%\end{array}$ & $\begin{array}{c}21 \\
0: 03: 59 \\
10.0 \%\end{array}$ & $\begin{array}{c}21 \\
0: 03: 52 \\
11.8 \%\end{array}$ & $\begin{array}{c}29 \\
0: 04: 12 \\
5.7 \%\end{array}$ & $\begin{array}{c}48 \\
0: 08: 13 \\
11,3 \%\end{array}$ & $\begin{array}{c}156 \\
0: 25: 56 \\
9.2 \%\end{array}$ \\
\hline $\begin{array}{c}\text { total unidades y } \\
\text { tiempo } \\
\text { analizado(1) }\end{array}$ & $\begin{array}{c}266 \\
1: 03: 13 \\
100 \% \\
\end{array}$ & $\begin{array}{c}164 \\
0: 39: 48 \\
100 \% \\
\end{array}$ & $\begin{array}{c}153 \\
0: 32: 49 \\
100 \% \\
\end{array}$ & $\begin{array}{c}349 \\
1: 13: 31 \\
100 \% \\
\end{array}$ & $\begin{array}{c}320 \\
1: 12: 27 \\
100 \% \\
\end{array}$ & $\begin{array}{c}1252 \\
4: 41: 48 \\
100 \% \\
\end{array}$ \\
\hline
\end{tabular}


La codificación de la unidades informativa (usamos el concepto de unidad informativa en vez del de noticia porque se ajusta más a la homogeneidad que requiere el análisis cuantitativo de la información televisiva) permite constatar cual es el caso al que se le destina más tiempo. En el 2008 el segundo al que le prestaron mayor interés todas las cadenas televisivas, fue el de la muerte de Ousmane Kote. La noche del 7 de septiembre de 2008 un joven inmigrante de origen senegalés fue asesinado en Roquetas de Mar, Almería, cuando intentó mediar en una disputa. Todas las cadenas usan la expresión "batalla campal" al darse la noticia y saltan a la palestra informativa con la imagen de un contenedor ardiendo, facilitada por un videoaficionado (imagen 1), de muy poca calidad, que dramatizan aún más el conflicto social y mostrando a la víctima con el torso desnudo en el sofá (imagen 2).

Imagen 1 - Contendores en llamas grabados por un videoficionado

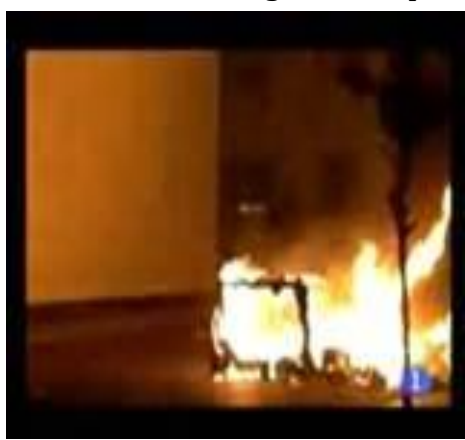

Fuente: LORITE (2011)

Imagen 2 - Fotografía difundida de Ousmane Kote

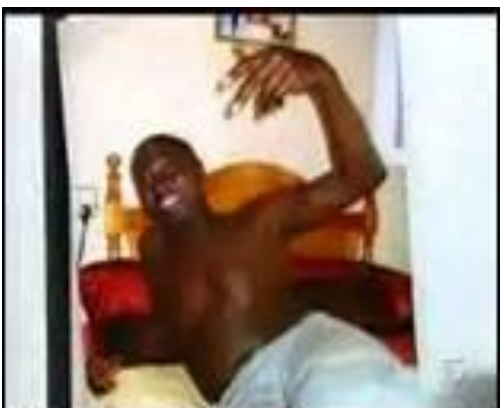

Fuente: LORITE (2011)

Todas las televisiones titulan "batalla campal". Hay una homogeneidad informativa total de partida. La televisión de cualquier signo le otorga un tratamiento bélico a un conflicto social entre ciudadanos de diferentes culturas. Al profundizar en el análisis vemos que dicho tratamiento inadecuado se da también al denominar a los protagonistas de la noticia. Al presunto asesino de Ousmane Kote se le llama de las siguientes maneras: "[...] la víctima "recibió una puñalada mortal de un vecino" (transmitido por el canal de televisión Antena3, 
07/09/08), "[...] se trataba de "un hombre de etnia gitana" (Cuatro, 07/09/08), " [...] fuentes de la investigación nos han dicho que saben que es español”(TVE 07/09/08), “[...] un vecino de etnia gitana" (TVG 07/09/08), "[...] al detener al asesino lo identifica como "el "Bollo", más conocido como "Juanjo el gitano"” (La Sexta, 22/09/08). En realidad descubrimos, al investigar el caso con la cámara para producir el documental, que se trataba de un hombre, no gitano, casado con una gitana.

Pero a la vez que vamos descubriendo qué hay tras dicha representación mediática asumimos otros roles y funciones propios de cualquier equipo de producción audiovisual. Pensamos, por ejemplo, cual debe ser la denominación adecuada de los protagonistas de la noticia y el espacio adecuado para plasmar la alternativa es el documental En tono a la muerte de Ousmane Kote (LORITE-GARCÍA; 2010). Todos los protagonistas que aparecen son tratados de la misma manera (LORITE-GARCÍA; GRAU REBOLLO, 2013).

Paralelamente también vamos determinando la tipología de documental y los medios y/o ámbitos de difusión, la función de la voz en off (decidimos suprimirla, se le da prioridad al discurso con las respuestas entrelazadas de los entrevistados), no usamos guion, sólo usamos escaleta, cuidamos la composición visual (prioridad del plano medio de los entrevistados en un espacio propio habitual, cámara frontal, estática, sin panorámicas ni traveling, considerando la regla de los tercios y en particular los cuatro puntos óptimos de información del cuadro), tenemos en cuenta también la narrativa con la cámara (MILLERSON, 1993), los entrevistados no miran a cámara, miran a un hipotético entrevistador situado al lado de la cámara, y el ritmo audiovisual (se tiene en cuenta la respuesta breve y para ello se les instruye previamente a los entrevistados en el modelo de montaje audiovisual que debe ejecutarse para facilitar el montaje posterior). Algo fundamental es la estructura narrativa del documental. Se fragmenta en once secuencias, pensada para su visionado íntegro o parcial. Cada secuencia tiene significado por sí misma. Se abordan por este orden las siguientes temáticas: información televisiva sobre el caso, contextualización socio-urbana de la víctima, lo que albergan en la memoria los vecinos sobre el conflicto, la cobertura inicial con la "batalla campal" difundida por todas las televisiones, los errores que se dan al denominar a los personajes, cómo aparecen las fuentes informativas, cómo tratan a los dos protagonistas principales (en concreto lo de mostrar a Ousmane Kote con el torso desnudo y el uso de las imágenes del videoaficionado del contenedor quemado), y una secuencia final con propuestas adecuadas para representar las relaciones interculturales en la televisión. Igualmente se tiene en cuenta la duración del documental: 42 minutos y 30 segundos (no se adapta a for- 
matos televisivos como el 30 o 60 minutos porque no está pensado para su difusión televisiva sino para un uso principalmente didáctico). También se determina editar con Adobe Premier y descartar AVID y Final Cut, usar sólo lo imprescindible de efectos (sólo se usan para realizar un montaje en la primera secuencia con las diferentes noticias sobre el caso), y se produce una música propia, diseñada por Eduardo Muraca tras llevar a cabo su análisis de las músicas de las sintonías de los telenoticiarios aunque sólo se usa los imprescindible: para poner el título del documental y con los créditos al final.

Es importante tener en cuenta la investigación cualitativa llevada a cabo con los protagonistas o fuentes informativas del documental. Se selecciona una muestra de entrevistados de acuerdo a los criterios metodológicos cualitativos de las ciencias sociales (GARCIA FERRANDO; IBAÑEZ; ALVIRA, 1994; OROZCO, 2000). Finalmente se llevan a cabo 26 entrevistas, realizadas a: los vecinos María Ruiz, y Francis (no quiso dar su apellido y optamos por ponerlo igualmente, aunque, según las recomendaciones oportunas, no debería aparecer si no tenemos el apellido, pero determinamos usar esta fuente porque era el único amigo del supuesto asesino); los políticos que intervinieron directamente en el caso (Gabriel Amat, alcalde de Roquetas de Mar, José Galdeano, concejal de Servicios Sociales, Juan Francisco Iborra técnico municipal de inmigración y trabajador del Área de Bienestar Social de Roquetas de Mar); los responsables de las organizaciones de inmigrantes (André Mendy de la Asociación Senegalesa de Roquetas de Mar, Ibrahima Sarr, presidente de la Asociación Senegalesa de Roquetas de Mar, Adama Sangare, mediador cultural), los periodistas y cámara (Antonio Torres, responsable de Canal Sur en Almería, Rocio Amores y Pablo Pumares, periodistas de Canal Sur, José Herrera, cámara de Canal Sur, Carles Solà, periodista de TV3, y Esteve Crespo editor del telenoticiario Noticias 2 de TVE) y otros expertos e investigador es como Ángeles Arjona, Antonio Bañón, María Trinidad Órtiz y Manuel Carretero de la Universidad de Almería, Eduard Bertran del MIGRACOM-UAB, Silvia Carrasco del Centro de Migraciones de la UAB, Dolors Comas y Rafael Jorba del Consejo del Audiovisual de Catalunya, Francisco Martín del Colegio Periodistas de Catalunya, Enric Prats de la Universidad de Barcelona, y Carlota Solé de la Universidad Autónoma de Barcelona (LORITE-GARCÍA; GRAU REBOLLO, 2013).

\section{Dinamización intercultural del discurso audiovisual}

Cómo pensamos un documental o mensaje audiovisual científico, que al ver visto pueda dinamizar las relaciones interculturales, partimos de una teoría sobre dinamización so- 
cial mediatizada de la que se resume a continuación lo esencial. Empieza a usarse en los ochenta con las radios locales y municipales catalanas (LORITE-GARCÍA, 1992, 2002, 2003, 2010) y se aplica posteriormente a los medios de comunicación en general y a cualquier discurso audio y/o visual. Consideramos que las relaciones sociales mediatizadas se estructuran en varios niveles de comunicación o rangos/peldaños. Se le aplica esta denominación porque se establecen de manera jerárquica (rangos) y escalonada (peldaños) de menor a mayor, en función del grado de dificultad. El rango/peldaño más elemental es el de la comunicación interpersonal mecánica, por encima, en dificultad, estará el de la comunicación intergrupal mecánica, más difícil aún será alcanzar la comunicación interpersonal activa y más compleja será la comunicación intergrupal activa. La comunicación interpersonal o intergrupal es mecánica cuando los interlocutores (sujetos o grupos) utilizan en sus diferentes actos comunicativos los códigos existentes y no los usan para generar otras respuestas comunicativas presentes y/o futuras en ellos mismos ni en los receptores de sus mensajes. Es el caso del televidente que ve una noticia y luego la comenta con familiar/es, amigo/s o vecino/s sin que dicho acto comunicativo transcienda de ese momento. Se trata de una dinámica comunicativa interpersonal e intergrupal bastante habitual. Se recibe información y se transmite a otras personas pero el discurso no propicia ningún tipo de respuesta activa. La comunicación interpersonal o intergrupal es activa cuando el visionado del discurso audiovisual dinamiza conscientemente el efecto comunicador y pretende emplazar e implicar a los receptores del mensaje a futuros encuentros comunicativos interpersonales o intergrupales. Es el caso de la persona que ve una información, comenta lo visto con alguien y dicho encuentro interpersonal transciende a una respuesta activa. El mensaje informa pero a la vez provoca un efecto dinamizador interpersonal que puede desembocar en un proceso comunicativo intercultural activo. Los televidentes se organizan y sugieren alternativas al margen de su recepción televisiva. Cada uno de estos niveles de comunicación interpersonales e intergrupales, mecánicos y activos, se subdividen en subrangos/subpeldaños, establecidos, al igual que los rangos/peldaños, de manera jerárquica y escalonada, y de menor a mayor en función del grado de dificultad que entraña establecerlos en un contexto social determinado. El escalonamiento de los subrangos/subpeldaños viene dado por los diferentes usos de la comunicación verbal y no verbal. Oscilaremos desde verbal sistemática y no verbal ocasional, pasando por verbal sistemática ocasional y no verbal, sistemática sólo verbal, sólo sistemática no verbal, verbal y no verbal ocasionales, sólo verbal ocasional y no verbal ocasional. Es imprescindible disponer, a partir de dicha división jerárquico- 
comunicativa, de unas reglas de medición cuantitativas y cualitativas de los niveles de comunicación. Al conocer dónde se ubican dichas tendencias comunicativas mediatizadas podemos comprender en que niveles se sitúan los procesos comunicativos interpersonales e intergrupales mediatizados por nuestro documental o por cualquier discurso audiovisual de cualquier medio. No obstante, dichas informaciones sólo nos permitirán atisbar la tendencia comunicativa principal en un momento determinado. Para comprender el impacto dinamizador intercultural real hay que tener en cuenta la evolución de los procesos de dinamización social mediatizados a lo largo de series temporales. Por tanto, no basta con saber cómo dinamiza por ejemplo la televisión informativa las relaciones interpersonales e intergrupales de manera activa o mecánica en un momento concreto sino a lo largo de diferentes momentos. Para ello hay que tener en cuenta los diferentes momentos equidistantes que separan cada observación. El calendario anual puede ser una herramienta idónea pero también lo son otros calendarios relacionados con las programaciones televisivas. Los procesos de dinamización social mediatizados se podrán conocer de manera cuantitativa en un momento concreto de una serie temporal al evaluar las tendencias dinamizadoras de los telespectadores en cada rango/peldaño. Pero también podremos detectarlos en función de los cambios observados en la serie temporal preestablecida. Tanto en un modelo como en otro debemos encontrar la principal tendencia o punto medio de dinamización. Al unir los puntos medios de dinamización aparecen las diferentes trayectorias dinamizadoras que nos permiten comprender si son crecientes, decrecientes y estables entre los diferentes niveles de comunicación establecidos (rangos/peldaños y subrangos/subpeldaños). No hay que olvidar otorgarle un valor objetivo, extraído desde diferentes criterios comunicativos (lingüísticos, políticos, ideológicos, culturales, etc.) a las tendencias crecientes, decrecientes o estables observadas, que permita evaluarlas objetivamente Es posible que la tendencia dinamizadora intercultural sea activa para unos colectivos pero tan sólo mecánica para otros. Para unos la interculturalidad y el mestizaje, dinamizados por la televisión informativa, pueden ser idóneos, para otros no. Por eso los procesos objetivos emergen de la similitud de las diferencias.

\section{Conclusiones con nuevas propuestas}

Este último apartado de conclusiones va destinado a agregar algunas pinceladas sobre cómo se traslada la experiencia investigadora audiovisual acumulada con los anteriores 
proyectos a los que trabajamos actualmente sobre la representación de la diversidad sociocultural en la publicidad y sus efectos dinamizadores interculturales.

Al igual que los otros proyectos procuramos estudiarlos en diferentes ámbitos mediáticos: catalán, español, internacional. A nivel de Catalunya profundizamos en la televisión catalana TV3 y analizamos la publicidad emitida durante los dos periodos del año 2014 siguiente: del 15 de enero al 15 de febrero, y del 15 de junio al 15 de julio. A nivel de toda España tenemos en cuenta las cadenas televisivas de ámbito estatal: TVE, Tele5, A3, Cuatro, La Sexta, y la autonómica catalana TV3. A nivel estatal grabamos, codificamos y analizamos los anuncios emitidos en horario de prime time (un horario amplio de cuatro horas que empieza a las 20,30 y acaba a las 0,30 horas), durante un semana de marzo de 2014. El proyecto $\mathrm{i}+\mathrm{d}+\mathrm{i}$ está financiado por el Ministerio de Economía y Competitividad y se titula "Estudio multimodal de la representación de la diversidad en la publicidad española y efectos interculturales en las ciudades del Mediterráneo en tiempos de crisis". Esta perspectiva de investigación audiovisual adquiere una dimensión comparativa internacional con el estudio "Publicidad, propaganda, alteridad y ciudadanía: estrategias transmetodológicas de análisis de la diversidad en contextos de cambio económico y social en Brasil y España", aprobado por Coordenação de Aperfeiçoamento de Pessoal de Nível Superior (CAPES), Brasil y el Ministerio de Educación español, en enero de 2015 y co-dirigido por Jiani Bonin (UNISINOS) y Nicolás Lorite (UAB).

En los tres estudios se persiguen validar planteamientos hipotéticos como los siguientes: "La publicidad no ha sabido integrar la diversidad que viene formándose en el tejido social español a partir de las migraciones transnacionales iniciadas a mediados de los 90, y continua (re)produciendo una falsa imagen tópica y estereotipada, asociada con ciertos fenotipos, tradiciones y costumbres principales de sus culturas de origen, que imposibilita desarrollar modelos de dinamización intercultural integradores o como mínimo normalizadores de las relaciones entre población autóctona e inmigrante y puede suscitar efectos racistas y xenófobos" (investigación de España). "La publicidad y la propaganda española y brasileña no han sabido incorporar la alteridad y la diversidad sociocultural existente en sus respectivos países y (re) producen una falsa imagen, tópica y estereotipada, construida con tan sólo algunos fenotipos e imposibilita desarrollar modelos de dinamización intercultural integradores y suscita efectos racistas y xenófobos" (investigación Brasil-España).

En los tres se usa una metodología cuanti-cualitativa, siempre del cuanti al cualitativo para analizar los anuncios televisivos. Las variables a tener en cuenta son: gru- 
po/nombre/marca (por ejemplo: Renault / Dacia / Sandero o bien L'Oreal / Garnier / Fructis), la duración en minutos y segundos de cada anuncio, la tipología del producto (limpieza, hogar, belleza e higiene, finanzas, alimentación, bebidas, vestimenta, salud, automoción, cultura, enseñanza y medios de comunicación, deporte, ocio y tiempo libre, cultura, arte y espectáculos, energía, decoración, telecomunicaciones e Internet, etc.), una sinopsis sobre lo que plantea y una clasificación de los anuncios en los que aparecen personas y en los que no. A partir de aquí se profundiza el análisis cualitativo en aquellos anuncios que representan actores diversos. Previamente establecemos un marco teórico en el que se delimitan los conceptos oportunos. Es esencial definir qué entendemos por diversidad sociocultural. El concepto utilizado en estas investigaciones audiovisuales se valida a partir de los primeros análisis, por parte de equipos interdisciplinares, de la muestra piloto de anuncios. Se huye de la incorporación de conceptos clásicos, universales. Se diseña no propio, adecuado a la investigación audiovisual. A partir de aquí analizamos los protagonistas diversos de los anuncios con actores. En concreto observamos el papel (principal, secundario o casual) que desempeñan los actores de otras culturas u orígenes y los autóctonos. Además tenemos en cuenta género, edad, complexión, estatura, clase social, contexto social, color de piel, color de ojos, cabello (tipo, color, longitud), fenotipo, origen, manera de vestir, uso de ropa occidental y tipo de interacción entre ellos. También se analizan los lenguajes textuales, gráficos, sonoros, visuales y audiovisuales de los anuncios con diversidad. En concreto, sobre el lenguaje visual tenemos en cuenta la ubicación y duración del plano, la composición visual, el tipo de plano y su valor y otros elementos como luz, color, transiciones visuales, ritmo, movimientos interno y externo al cuadro y tipo de montaje; sobre el lenguaje sonoro tenemos en cuenta la tipología de sonido (si es una voz en off, voz del personaje, música, efectos, silencios), el sonido diegético y extradiegético, el uso de la voz en off (lo que dice, género, edad, tono, idioma, acento), y la música, distinguimos si es clásica, popular, folclórica o bien de otro tipo, agregamos a todo ello si destaca un instrumento principal en la música y en caso de tener letra la canción transcribirla y adivinar a quien pertenece esa música.

Como se trata del estudio de la representación de la diversidad sociocultural en la publicidad lo hacemos desde la perspectiva multimodal procuramos obtener el máximo de referencias posibles desde la producción-emisión-recepción-dinamización de los mensajes o anuncios publicitarios en este caso. En el estudio de España pretendemos conocer desde la producción el proceso de selección de los actores en las agencias de casting, pero también qué imagen detentan los creativos sobre la población local o autóctona y la inmigrante, en 
qué se basan para distinguir una mujer española de una latina, africana u oriental. Desde la recepción del mensaje procuramos detectar, mediante una encuesta, pasada a universitarios de diferentes ámbitos del conocimiento (publicidad, comunicación audiovisual, antropología, pedagogía, periodismo principalmente), y mediante debates, sus opiniones sobre ciertos anuncios con diversidad, obtenidos en las muestras seleccionadas, como por ejemplo de qué color ven la piel de ciertos protagonistas y si el fenotipo lo asocian con alguna cultura, origen geográfico o país en particular. También es fundamental saber si consideran el anuncio como buena práctica publicitaria para suscitar la dinamización intercultural activa o lo ven como un mensaje capaz de producir efectos racistas y xenófobos. Todo ello nos lleva a producir paralelamente el discurso audiovisual alternativo y el documental científico que muestra los resultados de la investigación de una manera pedagógica para educar a los usuarios en la gramática audiovisual y en la formulación de la crítica y la propuesta alternativa desde el audiovisual también.

\section{Referencias}

ACHUTTI, Luiz Eduardo Robinson. Fotoetnografia: um estudo de antropologia visual sobre cotidiano, lixo e trabalho. Porto Alegre: Palmarinca, 1997.

ANDREW, John Dudley. Las principales teorías cinematográficas. Barcelona: Gustavo Gili, 1978.

BERGER, John. Mirar. Barcelona: Gustavo Gili, 2001.

BERGER, John. Modos de Ver. Barcelona: Gustavo Gili, 2000.

BERGER, John; MOHR, Jean. Otra manera de contar. Murcia: Mestizo A.C., 1997.

BERGER, Peter L.; LUCKMANN, Thomas. La construcción social de la realidad. Buenos Aíres: Amorrortu, 1984.

BERTRAN, Eduard. Información visual y migración. 2003. Tesis Doctoral (Doctorado de Comunicación Audiovisual y Publicidad). Facultad de Ciencias de la Comunicación. Universitat Autònoma de Barcelona. Policopiado, 2003.

CANEVACCI, Massimo. Antropología da comunicação visual. São Paulo: DP\&A Editora, 2001.

CHALMERS, Alan F. ¿Qué es esa cosa llamada ciencia?. Madrid: Siglo XXI, 2000.

CONSELL DE L'AUDIOVISUAL DE CATALUNYA. Manual sobre el tratamiento informativo de la inmigración en los medios de comunicación. Barcelona: Generalitat de Catalunya, 
2004.

FEYERABEND, Paul. Adiós a la razón. Madrid: Tecnos, 1984.

GARCIA FERRANDO, Manuel; IBAÑEZ, Jesús; ALVIRA, Francisco. El análisis de la representación social: métodos y técnicas de investigación. Madrid: Alianza, 1994.

GRAU REBOLLO, Jordi. Antropología Audiovisual. Barcelona: Bellaterra, 2000.

GRAU REBOLLO, Jordi. El document etnogràfic audiovisual generat en un projecte de recerca: proposta de projecte. Revista d'Etnologia de Catalunya, [S.l.], n. 23, p. 120-135, 2003.

GUBERN, Román. La mirada opulenta. Barcelona: Gustavo Gili, 1987.

IBAÑEZ, Jesús. Más allá de la sociología. Madrid: Siglo XXI, 1979.

KUHN, Thomas. La estructura de las revoluciones científicas. México: FCE, 1975.

LORITE-GARCÍA, Nicolás. Dinamización social y radio municipal. Tesis doctoral (Doctorado de Ciencias de la Información). Universidad Autónoma de Barcelona (UAB),Bellaterra, 1992.

LORITE-GARCÍA, Nicolás (dir). Tractament de la immigració no comunitaria en premsa, ràdio i televisió a Catalunya. Barcelona: Departament de Benestar Social, Generalitat de Catalunya, 1996.

LORITE-GARCIA, Nicolás. Medios, inmigración y dinamización intercultural: algunas propuestas para la investigación-acción desde el MIGRACOM. In: GARCIA CASTAÑO, F. Javier; MURIEL LÓPEZ, C. (Eds.). La inmigración en España: contextos y alternativas. Granada: Laboratorio de Estudios Interculturales, 2002. p. 449-456

LORITE-GARCÍA, Nicolás. Usages sociaux des NTIC et processus de dynamisation interculturels en Catalogne. In: LACROIX, J-Guy;TREMBLAY, Gaetan (Dir.). 2001 Bogues. Globalisme et pluralismo. Laval: Les Presses de l'Université Laval, Collection Éthique et philosophie de la communication, 2003.

LORITE-GARCÍA, Nicolás. Tratamiento informativo de la inmigración en España 2002. Madrid: Instituto de Migraciones y Servicios Sociales, 2004.

LORITE-GARCÍA, Nicolás. ¿Puede ser científica y objetiva la mirada audiovisual de la realidad migratoria?. In: LARIO BASTIDA, Manuel (Coord.). Medios de comunicación e inmigración. Murcia: Programa CAM encuentro, 2006a. pp. 85-96.

LORITE-GARCÍA, Nicolás. La mirada multipolar de la inmigración desde la perspectiva de la investigación audiovisual aplicada. Media e Jornalismo, Coimbra, n. 5, p. 35-54, 2006b. 
LORITE-GARCÍA, Nicolás. Metodologías de uso de los medios y lenguajes audiovisuales para el conocimiento de las realidades migratorias. In: BAÑóN, Antonio (Ed.). Discurso periodístico y procesos migratorios. Gakoa: Donosita-San Sebastián, 2007a. p. 117-138.

LORITE-GARCIA, Nicolás (Dir.). Tratamiento Informativo de la Inmigración en España: año 2007. Madrid: OBERAXE-MTIN, Bellaterra: MIGRACOM-UAB, 2007b.

LORITE-GARCIA, Nicolás (Dir.). Tratamiento Informativo de la Inmigración en España: año 2008. Madrid: OBERAXE-MTIN, Bellaterra: MIGRACOM-UAB, 2008a.

LORITE-GARCÍA, Nicolás. Discurso, inmigración y medios audiovisuales. In: BAÑON, Antonio; FORNIELES, Javier (Coords.). Manual sobre Comunicación e Inmigración. Gakoa: Bilbao, 2008b. p. 181-194.

LORITE-GARCIA, Nicolás (Dir.). Tratamiento Informativo de la Inmigración en España: año 2008. Madrid: OBERAXE-MTIN, Bellaterra: MIGRACOM-UAB, 2009.

LORITE-GARCÍA, Nicolás. Televisión informativa y modelos de dinamización intercultural. In: MARTÍNEZ LIROLA, María (Ed.). Migraciones, discursos e ideologías en una sociedad globalizada: claves para su mejor comprensión. Alicante: Colectiva, 2010. p. 19-43.

LORITE-GARCÍA, Nicolás (Dir.). En torno a la muerte de Ousmane Kote. Documental Producido por MIGRACOM y CER-Migracions. Bellaterra: Universidad Autónoma de Barcelona (UAB). 42 minutos y 30 segundos. Color, 2010. .

LORITE-GARCÍA, Nicolás. Informative Treatment of Immigration and Intercultural Dynamics Of Spanish Mass Media. In:URETA, Ivan (Ed.). Media, Migration and Public Opinion: Myths and Prejudices and the Challenge of Attaining Mutual Understanding between Europe and North Africa. Bern: Peter Lang, 2011.p. 187-214

LORITE-GARCÍA, Nicolás; GRAU REBOLLO, Jordi. Investigación audiovisual de las migraciones y el tratamiento de la diversidad en los medios de comunicación: un estudio de caso. In: GRANADOS, Antolín (Ed.). Las representaciones de las migraciones en los medios de comunicación. Madrid: Trotta, 2013. p. 139-155

MIGRACOM. Tratamiento Informativo de la Inmigración en España: año 2010. Bellaterra: MIGRACOM-UAB, 2010.

MILLERSON, Gerald. Técnicas de realización y producción en televisión. Madrid: IORT, 1993.

MURACA, Eduardo. Procesos comunicativos de la música:el caso de los discursos sonoros de las sintonías musicales en los informativos televisivos españoles y la comunicación instrumentalizada mediatizada. (Trabajo de Investigación)- Doctorado de Comunicación Audiovisual y Publicidad, Facultad de Ciencias de la Comunicación, Universidad Autónoma de Barcelona, Barcelona, 2009.

MURACA, Eduardo. Diseño y comunicación de la pluralidad sociocultural en las sintonías musicales de los teleinformativos. Tesis Doctoral (Doctorado de Publicidad y 
Relaciones Públicas)-Facultad de Ciencias de la Comunicación, Universidad Autónoma de Barcelona, Barcelona, 2013.

OROZCO, Guillermo. La investigación en comunicación desde la perspectiva cualitativa. Guadalajara: Universidad Nacional de La Plata, 2000.

POPPER, Karl. La lógica de la investigación científica. Madrid: Ed.Tecnos, 1962.

SENDÍN, José .Carlos; IZQUIERDO, Patricia. Guía práctica para los profesionales de los medios de comunicación: tratamiento informativo de la inmigración. OBERAXE, 2007. WATZLAWICK, Paul. ¿Es real la realidad?. Barcelona: Herder, 1995.

\section{A câmera como ferramenta principal para a pesquisa audiovisual dos processos de dinamização intercultural mediatizados}

\section{Resumo}

Este texto pretende mostrar as diferentes ideias sobre a câmera como principal ferramenta metodológica de pesquisa sobre o tratamento midiático da realidade, em particular, a reapresentação social nos meios de comunicação, realizadas pelo MIGRACOM (Observatório e Grupo de Investigação de Migração e Comunicação) da Universidade Autônoma de Barcelona (UAB), durante as duas últimas décadas (1996-2015). Se utiliza a câmera a partir de estudo interdisciplinar (comunicação, antropologia, educação, política, sociologia e linguística) do mesmo objeto de estudo audiovisual e a multimodalidade metodológica ou análise transversal da produção, transmissão, recepção e revitalização das mensagens audiovisuais. A câmera também permite que se vincule a investigação científica, acadêmica, com a verdadeira produção audiovisual. Seu uso emerge a partir da mistura de racionalidade quantitativa com a espontaneidade de todos os dias.

\section{Palabras-chave}

Câmera. Audiovisual. Televisão. Multimodalidade. Interculturalidade. 


\title{
The camera as a main tool for audiovisual research of mediatized intercultural dynamization processes
}

\begin{abstract}
This paper tackles different ideas about the camera as the main methodological tool to analyse media treatment of reality and particularity for a social re-presentational analysis within mass media, as it has been carried out by the MIGRACOM (Observatory and Research Group on Migration and Communication) at the Universitat Autònoma de Barcelona (UAB) for the two last decades (1996-2015). During that time, we have strongly supported the addition of criteria as in an interdisciplinary study (communication, anthropology, pedagogy, politics, sociology and linguistics) of the same audiovisual enquiry, the methodological multimodality or transversal analysis from complementary viewpoints concerning audiovisual messages: production, broadcasting, reception and dynamisation, as well as the necessary bond to scientific and academic research with real audiovisual production, thus thinking in the mixture of quantitative rationality and the spontaneity.
\end{abstract}

\section{Keywords}

Camera. Audiovisual. Television. Multimodality. Intercultural.

Recebido em 14/10/2015

Aceito em 11/12/2015 